\title{
Session 10: New approaches
}

Friday 14th November 2008. Moderator: Mark Glassy

\section{[14.00-14.30]}

Therapeutic cDNA vaccines and disease-specific monoclonal antibodies, which target CD44 on cancer and inflammatory cells

David Naor

The Lautenberg Center for General and Tumor Immunology, The Hebrew University - Hadassah Medical School, Jerusalem 91120, Israel

Synovial fluid cells (SFCs) of rheumatoid arthritis (RA) and psoriatic arthritis (PSA) patients express a CD44 variant (designated CD44vRA), which neither expressed on blood leukocytes of the same patient nor on synovial fluid cells of osteoarthritis patients and keratinocytes of normal donors (our article in JCI,111:121,2003). We produced anti-CD44vRA monoclonal antibodies that exclusively kill by apoptosis SFCs of RA and PSA patients and generate diseasespecific resistance in mice with collageninduced arthritis, implying the therapeutic feasibility of our antibodies. In addition, we showed that active generation of anti-CD44 antibodies (directed against CD44 variants) by CD44 gene vaccination markedly reduced the pathological activities in animal models of experimental autoimmune encephalomyelitis (EAE), type 1 diabetes and breast cancer.

\section{[14.30-14.50]}

New applications for rapid discovery of high value hybridomas and stable therapeutic antibody producing cells using ClonePix FL

Christopher J. Mann

Queensway, New Milton, Hampshire, BH25 5NN, UK

Tel.: +44 1425624617 ;

E-mail: chris.mann@genetix.com

ClonePix FL is widely used in the pharmaceutical and biotechnology sectors to rapidly find and isolate the best clones from large heterogeneous cell populations. This hunt for the right cell candidate is fundamental at both the discovery stage (finding antigen-specific clones from hybridoma fusions) and the development stage (isolating the highest producers from transfections). The power of ClonePix FL technology lies in its ability to simultaneously visualise and quantify the antibody or protein secreted from thousands of clones, and to select only the highest value candidates thus bypassing the need for high throughput automation.

In this presentation, new applications will be illustrated using case studies undertaken in collaboration with pharmaceutical and biotechnology companies. In one study, antigen-specific IgG-secreting hybridoma clones were selectively isolated from fusions within 8 days eliminating the need to collect and process large numbers of clones. The selectively picked clones were $89 \%$ positive by antigen-specific ELISA while remaining clones were $>99 \%$ negative. The need to fluorescently conjugate the detection antigen was bypassed by detecting a tag on the antigen. In a second study, hybridoma clones producing phosphopeptide-specific IgG were selectively isolated from a mixed population. Furthermore, the selected clones produced greater than 5 times more $\operatorname{IgG}$ than the best clones isolated when collected randomly. In a third study, the best clones secreting a monomeric therapeutic protein were screened from a transfected $\mathrm{CHO}$ population by using a fluorescently-conjugated probe against an expressed epitope tag. In a fourth study, an easy-to-use application was developed to provide an early indication of clone stability by measuring the tightness of antibody secretion from daughter clones.

[14.50-15.10]

The high affinity and potent XOMA 052 anti-IL-1 $\beta$ antibody: designed with a goal in mind Linda Masat XOMA (US) LLC, Berkeley, CA, USA

Through a series of strategic cross-licensing relationships and internal innovation, XOMA has created a premier Ab technology platform. It's comprised of six commercial human $\mathrm{Ab}$ phage display libraries for $\mathrm{Ab}$ 
discovery, proprietary Human Engineering(TM) technology, optimization technologies, and an integrated product development infrastructure. In addition to access to commercial Ab libraries, XOMA is enhancing the discovery platform by designing and constructing custom phage display Ab library from naïve as well as focused, patient-derived repertoires. The rationale for the custom libraries as well as their potential use for internal or collaboration programs will be presented. The success of XOMA's approach to Ab discovery and development is illustrated with XOMA 052, a 300fM anti-IL-1beta mAb currently in Phase I clinical testing. XOMA 052 preclinical in vitro and in vivo data will also be presented with potential clinical indications discussed.

\section{[15.10-15.30]}

\section{VelocImmune: A novel platform for human antibody discovery \\ Sean Stevens \\ Regeneron Pharmaceuticals Inc., Tarrytown, New York, USA}

VelocImmune mice are a novel platform for the rapid discovery of antibody therapeutics using the natural mouse immune system. The VelocImmune mice were created using Regeneron's VelociGene technologies to precisely replace germline variable regions of the mouse immunoglobulin loci (heavy chain V, D, and $\mathrm{J}$ segments, and light chain $\mathrm{V}$ and $\mathrm{J}$ segments) with the corresponding human germline variable sequences, leaving the mouse constant regions intact. Therefore, all murine antibody effector functions, such as Fc receptor and complement binding, are also retained.

Extensive analysis shows that the immune systems of VelocImmune mice are indistinguishable from wild type mice. VelocImmune mice have normal cell populations at all stages of B cell development in the bone marrow and in the periphery, exhibit normal rates of variable segment usage and somatic hypermutation and appropriate class switching. Furthermore, the responses to antigens by VelocImmmune mice are equivalent to wild type mice, and have generated monoclonal antibodies to a variety of antigens.

Multiple antibodies to important targets have been isolated, validated, and developed, leading to Regeneron's first fully-human antibody targeting the interleukin 6 receptor currently in clinical trials for the treatment of rheumatoid arthritis. Therefore, VelocImmune mice have been demonstrated to be an efficient platform for the rapid discovery of antibody therapeutics.

\section{[15.30-16.00]}

Thirty years of human monoclonal antibodies: Past, present and future developments Michael Steinitz

The Department of Pathology, The Hebrew

University-Hadassah Medical School, Jerusalem, 91120, Israel

E-mail: michaels@md.huji.ac.il

The idea of making human monoclonal antibodies emerged in the laboratory of George Klein in Stockholm independently of Köhler and Milstein's ingenious discovery. The idea was inspired by the amazing finding in the early seventies that in vitro, EpsteinBarr virus efficiently immortalizes human B cells giving rise to immunoglobulin-secreting lymphoblastoid cell lines. The significant advantages and potential of human monoclonal antibodies for passive immunization were exciting: they would resemble the very same antibodies produced in vivo to cope with the pathogen/assaulting factors and, if injected into patients, they would not be rejected. Very similar to the murine system, the essential prerequisites for making human monoclonal antibodies were a) accessibility to antigen-committed B cells and b) a consistent and reliable method to immortalize these cells. Practically, peripheral blood derived from immune humans is the sole accessible source of antigen committed B cells, the later being normally represented at a very low frequency. To overcome these difficulties, sensitive methods were improved to select antigen-committed B cells, and in vitro and murine in vivo systems were developed to sensitize naïve human lymphocytes.

Later on, molecular techniques were applied to engender partly or fully human monoclonal recombinant antibodies. These techniques enabled even production of antibodies with specificities that did not exist in the B cell repertoire of the donor of lymphocytes.

Production of human monoclonal antibodies was initially and primarily intended for passive immunization of patients with acute or chronic bacterial/viral infection and also for the treatment of cancer patients. Some monoclonal antibodies used now in the clinic as passive vaccines are clearly more beneficial compared to the non-specific Intravenous Immunoglobulin reagent (IVIG) given today to patients with a variety of pathologies.

There is a linkage between the presence of specific autoimmune antibodies and several pathologies of cognitive behaviour. Thus, the recent developments in immunotherapy of Alzheimer's disease (AD) focus on developing therapeutic human monoclonal antibodies 
against amyloid beta $(\mathrm{A} \beta)$, the protein which forms extensive plaques in the brain of the patients. This approach is supported by the findings, first, that anti$\mathrm{A} \beta$ antibodies are ubiquitously present in the sera of humans, thus presumably playing an important role in the homeostasis of $\mathrm{A} \beta$, and second, that cognitive improvement is induced in IVIG-treated AD patients. Recently we showed that anti-A $\beta$ antibody-secreting cell lines can be established from practically every healthy individual.

The association of certain psychiatric pathologies in some Lupus patients and the presence of specific autoimmune antibodies emphasize the possible cognitive effects of autoantibodies. We now try to generate non- hazardous human monoclonal antibodies against specific epitopes on the surface of neurons which would interfere with anti-neuron pathogenic antibodies.

Obvious difficulties which encountered the generation of human monoclonal antibodies have slowed down the development of the field compared to that of the murine. Today, molecular techniques and new revolutionary immunotherapies endorse intensive efforts to make indispensable human monoclonal antibodies.

[16.00]

Closing remarks

Mark Glassy - HAH 2008 Conference Chairman 